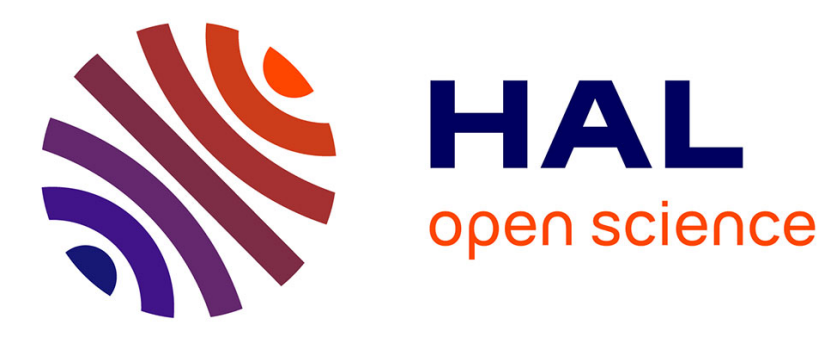

\title{
Shared control strategy for needle insertion into deformable tissue using inverse Finite Element simulation
}

\author{
Paul Baksic, Hadrien Courtecuisse, Bernard Bayle
}

\section{To cite this version:}

Paul Baksic, Hadrien Courtecuisse, Bernard Bayle. Shared control strategy for needle insertion into deformable tissue using inverse Finite Element simulation. ICRA 2021 - IEEE International Conference on Robotics and Automation, May 2021, Xi'an / Virtual, China. hal-03191078

\section{HAL Id: hal-03191078 \\ https://hal.science/hal-03191078}

Submitted on 6 Apr 2021

HAL is a multi-disciplinary open access archive for the deposit and dissemination of scientific research documents, whether they are published or not. The documents may come from teaching and research institutions in France or abroad, or from public or private research centers.
L'archive ouverte pluridisciplinaire HAL, est destinée au dépôt et à la diffusion de documents scientifiques de niveau recherche, publiés ou non, émanant des établissements d'enseignement et de recherche français ou étrangers, des laboratoires publics ou privés. 
(C)20XX IEEE. Personal use of this material is permitted. Permission from IEEE must be obtained for all other uses, in any current or future media, including reprinting/republishing this material for advertising or promotional purposes, creating new collective works, for resale or redistribution to servers or lists, or reuse of any copyrighted component of this work in other works. 


\title{
Shared control strategy for needle insertion into deformable tissue using inverse Finite Element simulation
}

\author{
Paul Baksic*, Hadrien Courtecuisse* and Bernard Bayle*
}

\begin{abstract}
This paper deals with the problem of needle steering in deformable tissues subject to physiological motions. A novel shared control method is proposed, which combines an automatic needle steering algorithm with the motions applied by the radiologist, in order to place the needle tip at the desired location. The core motivation is to leave potentially dangerous decisions and actions to the practitioner, whereas complex nonintuitive manipulations of the needle are performed automatically, in particular to compensate for breathing motions. The most original part of the present work lies in the method used to combine user inputs with a closed-loop automatic needle steering control method based on inverse Finite Element simulations. The method is evaluated with a realistic virtual environment using 2D $\mathrm{X}$-ray projection images. The results are compared with those obtained with a fully teleoperated system, on the one hand, and with a fully automatic solution, on the other hand. These experiments show that the shared control solution allows for a better needle tip placement when only projection imaging is available.
\end{abstract}

\section{INTRODUCTION}

Percutaneous image-guided interventions have become gold standard procedures for many medical treatments such as lung or liver tumor ablation, vertebroplasty, or nephrolithotripsy. Despite numerous advantages for patients, such procedures require advanced skills from the practitioner. In particular for percutaneous interventions involving particularly soft and mobile organs such as the liver. The difficulty lies in the complex tissue deformations due to needle-tissue interactions and physiological motions, in particular respiratory motions. Tissues deformations are difficult to anticipate for the practitioner, especially for deep liver tumors.

Imaging modalities used for percutaneous procedures usually depend on the tumor visibility, the practitioner preference and the local availability [1], [2]. Due to its low cost, its harmlessness and its real time capability, the most spread imaging modality is ultrasound. Yet, it is limited to superficial tissues and subject to artifacts due to air filled structures such as lungs or bowel [3]. Real time imaging such as CT fluoroscopy or real-time MRI is sometimes preferred for complicated cases [4], [5], [6]. However, due to the difficult access to the patient in MRI bores and to overexposure to $\mathrm{X}$-rays in CT fluoroscopy, the most commonly used imaging devices for percutaneous procedures into the liver remain conventional MRI and CT [4]. Using these, the insertions are done outside of the imaging device, and 3D images are only taken at key moments of the operation, but not in real time. This raises significant difficulties for needle guidance during the whole procedure.

\footnotetext{
*ICube Laboratory, UMR 7357, CNRS - University of Strasbourg, Strasbourg, France.
}

In the following we will focus on liver tumor ablation, for which the required tip positioning accuracy is $\approx 3$ $\mathrm{mm}$ [7]. This is therefore rarely obtained after the first needle insertion, and the practitioner usually needs to correct the trajectory several times, possibly stopping the patient breathing for a few seconds to limit disturbing motions. Such iterations involving successive needle insertion and removal increases the risk of tissue damages and the probability of spreading tumor tissues along the needle path while removing the needle. In order to overcome the aforementioned difficulties, robotic assistance has been proposed. Robots dedicated to percutaneous interventions can be classified into three different types, depending on their level of autonomy: manual, automatic and with shared control.

Robot for the assistance of interventional radiology are generally operated manually [8], being either teleoperated master-slave systems [9], [10] or even passive guides [11], [12], [13], [14]. A recent example of teleoperated system starting clinical trials is the ZeroBot developed by Hiraki et al. [10]. Another example is the DEMCON needle placement system [12]. The rotation of the needle guide is done automatically using CT imaging following a predefined straight path. Such manual systems may solve the problem of initial registration and planning [15], but do not take into account the needle-tissue interaction.

Automatic needle steering methods have the ability to compensate for such complex interactions (see [16] for a recent review) and therefore received significant interest in the last two decades. Methods have been proposed for both symmetric [17], [18], [19], [20], [21] and beveledtip needles [22], [20], [23], [24]. Chevrie et al. [23] relie on a needle/tissue interaction model combined with base manipulation and tip deflection to steer a beveled-tip needle. Similarly, Adagolodjo et al. [21] make use of inverse Finite Elements Models and needle-tissue interaction constraints to derive robotic commands for needle automatic steering. Though the use of models in these methods may increase the predictive nature of the tip position control, they require to consistently associate observed features with the underlying models. This makes them highly sensitive to registration errors, which are extremely difficult to compensate for peroperatively.

Shared control methods enable the user to be proactive during the task, and thus more responsive to errors [25]. Yet, due to the difficulty to merge user and automatic inputs, only few shared-control systems have been proposed for the needle steering inside soft tissue. Several authors [26], [27], [28] propose methods to steer beveled-tip needles. Chevrie et 
al. [28] give the user full control over the needle tip velocity through a haptic interface. The user feeds the desired tip velocity to the automatic needle steering method proposed in [23] that is used to control the needle. Wartenberg et al. [27] only give the possibility to the user to act on the insertion velocity, while the bevel orientation is obtained automatically.

The present paper introduces a robotic shared control method allowing for a needle steering into soft tissue. The contribution is built on the automatic method introduced by Adagolodjo et al. in [29] and later extended by coauthors of the present paper [30]. By giving the user control over the tip positioning objective function of the automatic algorithm, the proposed shared control method allows combining decision-making and automatic needle steering. The paper is organized as follows. Section $\Pi$ introduces previous work on which the contribution relies. Section III presents the contribution. Section IV details the parametrization of the method used in the experiments. Sections $\mathrm{V}$ and $\mathrm{VI}$ present and discuss the results of the experiments.

\section{Background: Automatic Needle Steering}

The understanding of the present paper requires to introduce the principles of the automatic method developed in [29], [30]. In these works, the needle insertion problem is expressed as the minimization of a cost function. The cost function is composed of multiple objective functions, allowing following a pre-defined path. In order to simplify the notations, each vector written without superscript is expressed in a global coordinate system that is supposed to be fixed with regard to the robot base.

Following [29], the path is a set of points interpolated by edge elements. At the beginning of the simulation, each point $\mathbf{q}_{t r a j}$ of the pre-defined trajectory is linked to the organ model using a barycentric mapping. This is done by expressing the coordinates of $\mathbf{q}_{t r a j}$ as a weighted sum of the coordinates of the points constituting the tetrahedron in which the point is located. If $l$ is the index of this tetrahedron, the barycentric coordinates of the point $\mathbf{q}_{t r a j}$ in the tetrahedron $l$ are $\left(\alpha_{1}, \alpha_{2}, \alpha_{3}, \alpha_{4}\right)$, such as:

$$
\mathbf{q}_{\text {traj }}=\sum_{i=1}^{4} \alpha_{i} \mathbf{q}_{l, i}
$$

With $\mathbf{q}_{l, i}$ the coordinates of the $i^{\text {th }}$ point of the tetrahedron $l$ and $\alpha_{i}$ is its associated barycentric coordinate.

The needle is supposed to be rigidly held by the end effector of the robot. The method provides at high frequency the displacement of the robot end effector resulting in needle tip motion along the path. Due to the coupling between the needle and the organ and potential contact with surrounding tissues, the relationship between the robot end effector position and the objective functions to be minimized for task completion is highly non-linear and non-holonomic. In order to minimize the non-linear cost functions, [29] proposed to use inverse Finite Element simulations to linearize the objective functions around the robot end-effector position.
The linearization of the objective functions consists in finding the so-called Jacobian of the simulation $\mathbf{J}^{(i)}$ linking the objective function evolution $\Delta \mathbf{e}^{(i)}$ to the robot endeffector motion $\Delta \mathcal{X}^{(i)}$ at a specific step $i$ :

$$
\mathbf{J}^{(i)} \Delta \mathcal{X}^{(i)}=\Delta \mathbf{e}^{(i)}
$$

This is achieved by perturbing each degree of freedom (DOF) $j$ of the robot end effector around its current position $\mathcal{X}^{(i)}$ in the FE simulations, and evaluating the resulting objective function evolution.

The Jacobian of the simulation is then used to compute a target $\mathbf{T}^{(i+1)}$ which is a linear solution reducing the objective function values:

$$
\mathbf{T}^{(i+1)}=\mathcal{X}^{(i)}-\mathbf{J}^{+(i)}\left(\mathbf{k} \odot \mathbf{e}^{(i)}\right)
$$

with $\mathbf{J}^{+(i)}$ being the pseudo-inverse of $\mathbf{J}$, $\odot$ is the Hadamard product and $\mathbf{k}$ is a gain vector allowing weighting the objective functions separately (see [30] for more precision).

The problem is solved computing the pseudo-inverse of the Jacobian combined with the Tikhonov regularization strategy in order to limit numerical instabilities. Finally, the target $\mathbf{T}^{(i+1)}$ is sent to the robot controller which solves the inverse kinematics problem allowing this way to move the robot such that the needle reaches the target on the trajectory.

The inverse simulation running at high frequency with regards to the velocity of the robot, this target is updated quickly enough to compensate for the non-linearity of the objective functions. The method was tested experimentally in [21] then optimized numerically in [30] allowing for the compensation of external perturbations in a realistic simulation of a pig liver moving under breathing motion and involving contact with surrounding tissues.

\section{METHODOLOGY}

\section{A. Justification}

An important limitation of the previous solution is that robot movements are generated if and only if a solution reducing the error is found for the linearized problem of equation (3). This implies that the trajectory is assumed to be accessible by moving only the base of the robot. Though the accessibility of the trajectory could be theoretically estimated by trajectory planning simulations, it is difficult to predict in practice because of the strain non-linearity and the mechanical interactions between the needle and the tissue. In addition, the accessibility of the trajectory may be affected by the insertion speed, which may itself vary depending on current tissues deformations and path curvature, which is difficult to adapt with the automatic solution. Finally, for responsibility and acceptance reasons it seems still necessary to let the practitioner have some control over the needle insertion, at least in combination with the robotic system.

An overview of the method is given by Fig. 11 To allow the practitioner to have control over the automatic needle insertion, the method is composed of two parts: the needle steering and the decision-making. The steering part is done by the automatic method designed to follow inputs provided 
by the user. Contrary to usual teleoperated systems, the user controls the needle tip position relative to the tissue, without considering complex motion such as breathing, whereas the automatic method is used to follow the user's input while taking complex needle-tissue coupling into account. In addition, haptic guidance is added to help the user follow a pre-planned path, without any need for compensation of the global motion of the system.

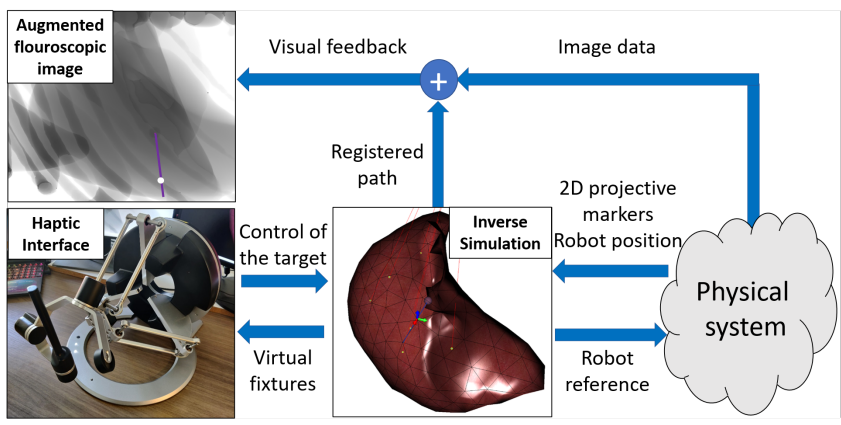

Fig. 1. Overview of the method. The user controls the needle tip placement during robotic percutaneous procedures through a haptic interface. Virtual fixtures are provided to guide him on a pre-defined path. An automatic needle-steering algorithm provides reference to the robot to follow the user's target, relying only on fluoroscopic images. Augmented fluoroscopic images representing the path are provided to the user as visual feedback, along with the registered 3D model of the organ.

Chevrie et al. [28] proposed a similar approach for beveled needles, in which the user controlled the Cartesian velocity of the needle tip. However, by relying on inverse FE simulations, and by linking the user input to the tissue through barycentric coordinates, the current solution enables to steer the needle to a specific point of the tissue while taking into account the needle-tissue coupling and external perturbations. Indeed, in [28], the steering algorithm allows controlling the Cartesian absolute velocity of the needle tip, which is therefore not relative to the tissue, and a fortiori neither to the aimed target. Only the effect of the needletissue coupling on the Cartesian tip velocity (through finite differences simulation) is taken into account.

\section{B. Automatic needle steering}

In order to define the Jacobian of the simulation $\mathbf{J}$ used for the automatic needle steering method, a set of objective functions must be defined:

1) Tip positioning: this objective function is a 3 dimensional vector $\mathbf{e}_{p}=\mathbf{q}_{\text {target }}-\mathbf{q}_{\mathrm{tip}}$, with $\mathbf{q}_{\text {target }}$ the position of the target and $\mathbf{q}_{\text {tip }}$ the position of the needle tip.

2) Needle orientation outside the patient: this objective function is a scalar $\mathbf{e}_{a}=\arccos \left(\mathbf{n}_{\text {tip }} \cdot \mathbf{n}_{\text {traj }}\right)$, with $\mathbf{n}_{\text {tip }}$ the normalized vector tangent to the needle at the tip and $\mathbf{n}_{\text {traj }}$ the normalized vector tangent to the trajectory at the current target point.

3) Pivot point at the insertion point: this function consists of a 2 dimensional vector $\mathbf{e}_{e}=\operatorname{proj}_{\mathcal{P}}\left(\mathbf{p}_{c}-\mathbf{p}_{p}\right)$. With $\operatorname{proj}_{\mathcal{P}}$ defined as the projection of a $3 \mathrm{D}$ vector on the plan $\mathcal{P}$ tangent to the insertion point in the skin. $\mathbf{p}_{c}$ is the current position of the insertion point and $\mathbf{p}_{p}$ the insertion position at the first penetration time.

When the needle is located outside the patient only objective function 1) and 2) are active whereas only 1) and 3) are used inside the body, resulting in a 4 or 5 dimensional error vector. The main difference with objective functions used in [30] is that the target position of the tip $\mathbf{q}_{\text {target }}$ is given by the user in the decision-making step.

\section{Decision-making}

A 6 Degrees Of Freedom (DOF) Omega 6 Force Dimension haptic device is used to provide the user full control over the tip positioning target $\mathbf{q}_{\text {target }}$. For this purpose, the visual feedback as well as the mapping between the user and the robot motion are both very important. In addition, virtual fixtures are added to help the user to follow the pre-defined path, while still being able to get away from it.

1) Visual feedback and registration: In order to steer the needle during the whole insertion, a real-time imaging modality is needed. For this purpose, 2D projective images are generated with X-ray rendering of virtual models ${ }^{1}$ Images shown to the user are therefore similar to the data acquired with fluoroscopy, lacking the 3D depth in the direction of the X-ray transmitter.

The registration of the model with $2 \mathrm{D}$ protective images is a complete research area that goes far beyond the scope of this paper. The current trend is to recover the $3 \mathrm{D}$ position of the model with neural networks approaches [31], but it still has to be demonstrated for abdominal organs. More importantly, the shared control method introduced in this article is completely independent of the registration strategy.

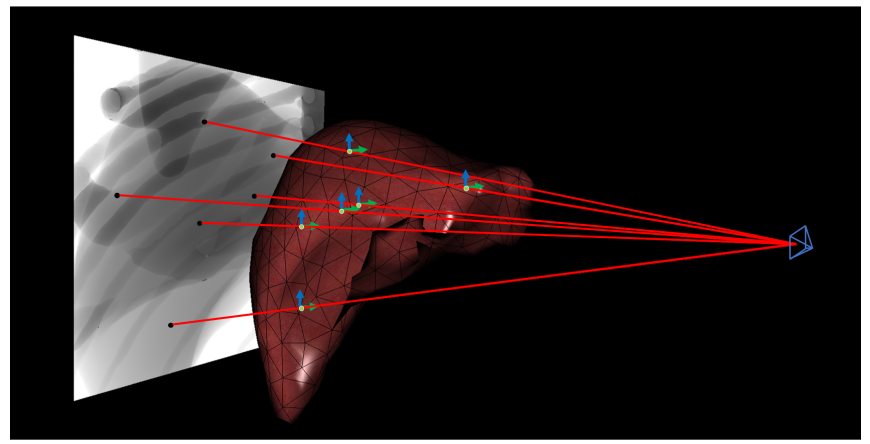

Fig. 2. Projective constraints. Registration of a liver using projective constraints. The imaging plane is represented (left) along with the position of the radiating source (in blue), the internal marker projections (black dots), and the resulting projection lines (red lines). To enforce registration, the $3 \mathrm{D}$ markers (green dots) are constrained on the projection lines using 2D projective constraints (blue and green arrows). The mechanical solver then finds the minimal energy position of the liver, enforcing the constraints.

In this work, the 3D position of a set of fiducial markers located in the volume of the liver is assumed to be known at the rest configuration (corresponding to the position of the model during the segmentation). In practice, fiducials may

\footnotetext{
${ }^{1}$ Images are generated using the open-source Sofa framework and an Xray rendering plugin available at: https://www.sofa-framework. org/applications/marketplace/xray-rendering/
} 
be inserted percutaneously before the CT-scan acquisition (later used for the segmentation), as performed by [32] to guide the Cyberknife. The registration of the model is then performed with $2 \mathrm{D}$ projective constraints as introduced in [33]: the markers are only constrained along the projective lines coming from the center of the projection of the C-ARM as shown in Fig. 2 Therefore the displacement of markers is prescribed in the $2 \mathrm{D}$ plane of the projected view but completely free in the orthogonal direction. The mechanical solver provides the $3 \mathrm{D}$ positions of the model minimizing the energy between projective constraints and internal forces of the model. After the registration, the 3D positions are used to both compute the Jacobian of the simulation $\mathbf{J}$ as explained in the previous section and display with augmented reality the medical data and the position of the registered path to the user, thanks to the aforementioned barycentric mapping (see equation (1)).

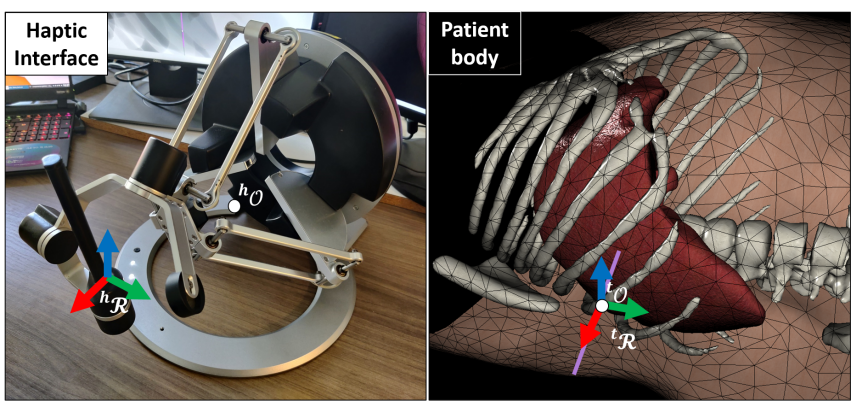

Fig. 3. Registration of the haptic interface with the current target $\mathbf{q}_{\text {target }}$ (white dot on the purple trajectory on the right-hand image). The $\mathbf{x}$ axis is represented in red, the $\mathbf{y}$ axis in green and the $\mathbf{z}$ axis in blue.

2) Master-slave mapping: As visible in Fig. 3, a local system of coordinates ${ }^{t} \mathcal{R}$ is attached to the target. Its origin ${ }^{t} \mathcal{O}$ is set at the position $\mathbf{q}_{\text {target }}^{*}$ of the target on the path. Its ${ }^{t} \mathbf{X}$ axis is defined as tangent to the trajectory, in the direction of the path starting point. The ${ }^{t} \mathbf{z}$ axis is first defined as collinear to the normal of the patient coronal plane, following the anterior direction. It is then set orthogonal to the ${ }^{t} \mathbf{x}$ axis following a Gram-Schmidt ortho-normalization algorithm. Finally, the ${ }^{t} \mathbf{y}$ axis is the cross product between ${ }^{t} \mathbf{z}$ and ${ }^{t} \mathbf{x}$. This coordinate system depends on the position $\mathbf{q}_{\text {target }}^{*}$ on the path and needs to be updated during the insertion.

The fixed coordinate system of the haptic interface ${ }^{h} \mathcal{R}$ is also represented in Fig. 3. Its origin ${ }^{h} \mathcal{O}$ is located at the center of the interface workspace. The ${ }^{h} \mathbf{x}$ axis of the haptic interface represents the advancement of the target along the path. As a result, a motion $\Delta x_{t}$ of the haptic interface endeffector along $-{ }^{h} \mathbf{x}$ moves the target position $\mathbf{q}_{\text {target }}^{*}$ forward along the path of a distance $\Delta x_{t}$. Moreover, in order to avoid involuntary motions, the user needs to press a button on the haptic end-effector to act on the target. Therefore, the button can be used as a clutch for the insertion depth control, to take maximum advantage of the interface working space. The position $(a, b)^{T}$ of the interface end-effector in the $\left({ }^{h} \mathbf{y},{ }^{h} \mathbf{z}\right)$ plane is added to the target following $\mathbf{q}_{\text {target }}=\mathbf{q}_{\text {target }}^{*}+$ $a^{t} \mathbf{y}+b^{t} \mathbf{z}$, this is then used as the tip positioning objective function target.

Finally, the target position is expressed as barycentric coordinates of the tetrahedrons composing the organ mesh. It is important to notice that since the user acts on the position of the target relative to the path position, which is linked to the tissue through barycentric mapping, the target $\mathbf{q}_{\text {target }}$ automatically follows the tissue motion and deformation. Therefore the user does not need to compensate for such disturbances and can concentrate on the needle insertion within the tissue.

3) Virtual fixtures: The insertion is guided by applying two types of virtual fixtures to the haptic device.

Along the ${ }^{h} \mathbf{y}$ and ${ }^{h} \mathbf{z}$ axes, a nonlinear spring-damper system is emulated to bring back the target on the predefined path. The force applied to the interface end-effector at a given position ${ }_{e e}^{h} \mathbf{q}=(x y z)^{T}$ of the haptic interface is computed as follows:

$$
\mathbf{f}_{s}=-\frac{{ }_{e e}^{h} \mathbf{q}_{y z}}{\left\|{ }_{e e}^{h} \mathbf{q}_{y z}\right\|} f_{\text {max }}\left|\operatorname{sigm}_{\omega_{s}}\left(\left\|\left.\right|_{e e} ^{h} \mathbf{q}_{y z}\right\|\right)\right|-b_{s}{ }_{e e}^{h} \dot{\mathbf{q}}_{y z}
$$

with ${ }_{e e}^{h} \mathbf{q}_{y z}={ }_{e e}^{h} \mathbf{q}-{ }^{h}\left(\begin{array}{lll}x & 0 & 0\end{array}\right)^{T}, f_{\max }$ the maximum allowed force, and $b_{s}$ the damping factor. The function :

$$
\operatorname{sigm}_{\omega_{s}}(d)=\frac{1-e^{-d \omega_{s}}}{1+e^{-d \omega_{s}}}
$$

is a sigmoid built in order to have the desired value $a_{s} \in\left[0,1\left[\right.\right.$ of the activation function at a distance $d_{s}$, with:

$$
\omega_{s}\left(d_{s}, a_{s}\right)=-\frac{1}{d_{s}} \ln \left(\frac{1-a_{s}}{1+a_{s}}\right)
$$

The virtual fixture is designed to guide the user on the predefined path leaving the freedom to get away from it. Close to the path (linear part of the sigmoid), the guide acts like a spring. After a certain distance is reached (asymptotic part of the sigmoid), the maximum force is reached and the user can move freely since only a constant force is applied to the interface in the direction of the predefined path.

The second virtual fixture emulates a damper system along the ${ }^{h} \mathbf{x}$ axis. Since the user controls the target needle tip position $\mathbf{q}_{\text {target }}$ and not directly the needle tip position, a delay between the user inputs and the needle steering may appear. It is therefore necessary to provide some feedback to the user to identify the actual position of the needle tip. This virtual fixture helps the user not to advance the target on the trajectory too quickly with regard to the steering velocity, which may cause the divergence of the automatic method. The applied force is computed as follows :

$$
\mathbf{f}_{d}=-{ }_{e e}^{h} \dot{\mathbf{q}}_{x} b_{\max }\left|\operatorname{sigm}_{\omega_{d}}(\|\mathbf{e}\|)\right|
$$

with ${ }_{e e}^{h} \dot{\mathbf{q}}_{x}$ the velocity of the end effector along the ${ }^{h} \mathbf{x}$ axis, $b_{\max }$ the desired maximum damping coefficient and $\mathbf{e}$ a vector composed of all the objective function values gathered in a vector, the norm of which is representative of the current error the automatic algorithm still needs to compensate given the current position of the target. 


\section{EXPERIMENTAL FRAMEWORK}

The method is evaluated by several users performing a simulated needle insertion to reach a tumor located at a depth of $60 \mathrm{~mm}$ within the liver $(100 \mathrm{~mm}$ deep under the patient's skin). For this purpose a direct simulation involving multiple deformable organs and contacts is implemented. This simulation represents the physical system shown in figure 1 in which the user has to place the needle. Based on this simulation, virtual X-ray images are generated and displayed to the users. The objective is to compare both the needle tip positioning accuracy and the insertion time in different cases. The shared-control method introduced in this article (SC) is compared with a fully teleoperated insertion (Man) on the one hand, and with a fully automatic one (Auto, detailed in [30]) on the other hand. Both the shared controlled and automatic solution rely on an inverse simulation running asynchronously to compute the commands of the robot holding the needle.

\section{A. Direct simulation}

The direct simulation is a realistic FEM simulation of the needle insertion procedure. Patient-specific simulated organs are the liver with a tumor, the diaphragm, the skin, and the rib cage, obtained by segmentation of real organs. Contacts between the liver and the diaphragm are simulated. Respiratory motion is obtained by applying a sinusoidal motion to upper points of the diaphragm mesh. By simulating the contacts between the diaphragm and the liver along with the effect of two ligaments placed on the back of the liver to constrain its motion, the liver follows the respiratory motion. This skin surrounding the insertion point is simulated and rigidly attached on two sides to simulate ribs. A simulated needle is rigidly attached to the robot end-effector. The robot end-effector is moved towards the target $\mathbf{T}$ at a constant velocity of $15 \mathrm{~mm} / \mathrm{s}$ and angular velocity of $0.2 \mathrm{rad} / \mathrm{s}$. The organs are simulated using a linear corotational formulation of the stress-strain relationship. All the model parameters are listed in Table 【.

\begin{tabular}{|c||c|c|c|c|c|}
\hline Model & $\begin{array}{c}\text { Young } \\
\text { modulus }\end{array}$ & $\begin{array}{c}\text { Poisson's } \\
\text { coefficient }\end{array}$ & $\begin{array}{c}\text { Number } \\
\text { of node }\end{array}$ & $\begin{array}{c}\text { Number of } \\
\text { element }\end{array}$ & $\begin{array}{c}\text { Type of } \\
\text { element }\end{array}$ \\
\hline Liver & $8 \mathrm{kPa}$ & 0.4 & 498 & 1999 & Tetra \\
\hline Diaphragm & $100 \mathrm{~Pa}$ & 0.3 & 60 & 24 & Hexa \\
\hline Skin & $10 \mathrm{kPa}$ & 0.3 & 216 & 750 & Tetra \\
\hline Needle & $200 \mathrm{GPa}$ & 0.3 & 14 & 13 & Edges \\
\hline
\end{tabular}

TABLE I

PARAMETRIZATION OF THE MECHANICAL MODELS.

Needle-tissue coupling is simulated using Duriez et al. [34] method, between the needle and the skin, the liver. Coupling constraints are placed every $8 \mathrm{~mm}$ inside both models. No friction inside the organs was considered.

A C-ARM is placed as shown in Fig. 2, corresponding to a sagittal view of the considered organ. It is used to compute the fluoroscopic rendering that is shown to the user, and for the liver registration in the inverse simulation.

\section{B. Inverse simulation}

As in [30], in order to minimize the solving time of the inverse simulation, only the needle and the liver (with the tumor), along with the needle-tissue interaction are simulated. The liver registration is done using 2D position of each marker, obtained from their 3D position in the direct simulation and the projection matrix of the C-ARM source.

\section{Virtual fixtures}

For SC insertions, the first (resp. second) virtual fixture is parameterized with $d_{s}=5 \mathrm{~mm}, a_{s}=0.98, b_{s}=10 \mathrm{Nm} / \mathrm{s}$ (resp. $d_{d}=0.001, a_{d}=0.98$ ). In order to study the effect of parameters $f_{\max }$ and $b_{\max }$, four different versions of $\mathbf{S C}$ were tested (see Table. II). SC $\mathbf{1 - 2}$ provides more lateral

\begin{tabular}{|c|c|c|c|c|}
\cline { 2 - 5 } \multicolumn{1}{c|}{} & SC 1 & SC 2 & SC 3 & SC 4 \\
\hline$f_{\max }(\mathrm{N})$ & 4 & 4 & 2 & 2 \\
\hline$b_{\max }(\mathrm{Ns} / \mathrm{m})$ & 120 & 60 & 120 & 60 \\
\hline \multicolumn{5}{c}{ TABLE II }
\end{tabular}

Parametrization of the SC METhods

guidance to the user to stay on the pre-defined path than SC 3-4. While a higher value of $b_{\max }$ along ${ }^{h} \mathbf{X}$ in SC 1 and $\mathbf{3}$ will reduce the amount of delay between the target and the tip position.

\section{Teleoperated insertion}

For Man insertions, the haptic interface is used for the teleoperation of the robot end-effector. The interface is registered, and its motions scaled, in order to allow the user to make the whole needle insertion in its workspace. The interface $\mathbf{x}$ axis is registered with the insertion path, and the $\mathbf{z}$ axis is oriented to the opposite of the gravity. The robot in the direct simulation is not constrained to ensure total transparency of the master-slave interface.

\section{E. User interface}

For the SC and Man experiments, the user is provided with a visual feedback, as proposed in section III-C.1. along with the registered model of the inverse simulation on another screen (see Fig. 47.

\section{F. User study}

A total of six untrained users and the system developer, denoted as the highly trained user, did the experiments. The users were asked to place the needle tip in the center of the tumor, without any limit of time. They were asked to make sets of five successive insertions of the same type. The users were given as much time as needed before each set to familiarize with the insertion type and move the interface.

All of the user did one set of Manu insertion, and either one or two SC sets (SC 1-4 randomly chosen). The highly trained user did two sets of Manu insertion and one of each SC. The orders of execution were randomized. Each of the insertions was executed until the user estimated that he could not place the needle tip better. 


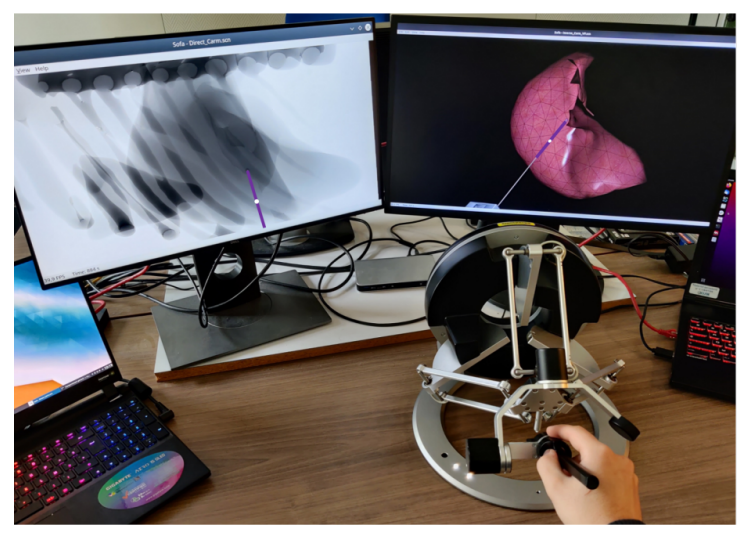

Fig. 4. Trials setup. The user is provided with augmented fluoroscopic image (left) and the registered liver inside of the inverse simulation (right).

\section{RESULTS}

Results of the user study are presented in table [III. The data set sizes are given in the last column. The trained user's results are given in bold. In-plane error stands for the error on the C-ARM projection plane whereas Out-of-plane stands for the error normal to this plane.

\begin{tabular}{|l||c|c|c|c|c|}
\cline { 2 - 6 } \multicolumn{1}{c|}{} & $\begin{array}{c}\text { Absolute } \\
\text { error }(\mathrm{mm})\end{array}$ & $\begin{array}{c}\text { In-plane } \\
\text { error }(\mathrm{mm})\end{array}$ & $\begin{array}{c}\text { Out-of-plane } \\
\text { error }(\mathrm{mm})\end{array}$ & $\begin{array}{c}\text { Insertion } \\
\text { time }(\mathrm{s})\end{array}$ & $\#$ \\
\hline Auto & $2.01 \pm 1.47$ & $1.81 \pm 1.3$ & $0.72 \pm 0.84$ & $97 \pm 25$ & 20 \\
\hline Man & $10.71 \pm 7.36$ & $4.5 \pm 4.23$ & $9.21 \pm 6.78$ & $127 \pm 96$ & 30 \\
& $\mathbf{5 . 5 3} \pm \mathbf{2 . 8 3}$ & $\mathbf{2 . 9 4} \pm \mathbf{1 . 9 6}$ & $\mathbf{4 . 5 8} \pm \mathbf{2 . 3}$ & $\mathbf{6 4} \pm \mathbf{2 9}$ & $\mathbf{1 0}$ \\
\hline SC 1 & $1.97 \pm 1.58$ & $1.17 \pm 0.88$ & $1.4 \pm 1.51$ & $59 \pm 11$ & 19 \\
& $\mathbf{0 . 9 2} \pm \mathbf{0 . 5}$ & $\mathbf{0 . 4 9} \pm \mathbf{0 . 2 7}$ & $\mathbf{0 . 6 6} \pm \mathbf{0 . 6 2}$ & $\mathbf{4 8} \pm \mathbf{6}$ & $\mathbf{5}$ \\
\hline SC 2 & $1.57 \pm 0.59$ & $1.16 \pm 0.47$ & $0.97 \pm 0.58$ & $53 \pm 12$ & 10 \\
& $\mathbf{1 . 0 2} \pm \mathbf{0 . 5 5}$ & $\mathbf{0 . 4 4} \pm \mathbf{0 . 2 0}$ & $\mathbf{0 . 8 3} \pm \mathbf{0 . 6 6}$ & $\mathbf{4 1} \pm \mathbf{3}$ & $\mathbf{5}$ \\
\hline SC 3 & $2.01 \pm 1.19$ & $1.47 \pm 0.85$ & $1.17 \pm 1.11$ & $82 \pm 30$ & 10 \\
& $\mathbf{1 . 5 5} \pm \mathbf{0 . 7 4}$ & $\mathbf{0 . 9 4} \pm \mathbf{0 . 6 9}$ & $\mathbf{1 . 1 1} \pm \mathbf{0 . 6 8}$ & $\mathbf{5 2} \pm \mathbf{5}$ & $\mathbf{5}$ \\
\hline SC 4 & $2.64 \pm 1.41$ & $2.07 \pm 1.12$ & $1.62 \pm 0.88$ & $94 \pm 32$ & 10 \\
& $\mathbf{0 . 8 8} \pm \mathbf{0 . 7 3}$ & $\mathbf{0 . 7 9} \pm \mathbf{0 . 6 7}$ & $\mathbf{0 . 3 4} \pm \mathbf{0 . 3 4}$ & $\mathbf{4 3} \pm \mathbf{4}$ & $\mathbf{5}$ \\
\hline
\end{tabular}

TABLE III

MEAN NEEDLE TIP PLACEMENT ERRORS AND STANDARD DEVIATIONS.

The results show that the untrained users divided by five the absolute placement error using any $\mathbf{S C}$ method compared to Man. The absolute precision is equivalent to the Auto insertion for SC 1,3 and better for SC 2, due to the in-plane placement. In contrary, the out-of-plane precision is worst for each SC method compared to the Auto method. Yet, thanks to the augmented visual feedback, all the users were able to compensate for lateral misplacement by readjusting the needle after the insertion, which is not possible with Auto.

The needle tip positioning absolute error of the highly trained user was lower than that of the untrained users, and he did the insertions quicker. He divided by two the absolute error with regard to the automatic placement, in the SC 1,2 and 4. This is again due to a better in-plane placement. The out-of-plane error is either equivalent $(\mathbf{S C} \mathbf{1 , 2})$ or worst (SC
3), except for SC $\mathbf{4}$ where the error is divided by a factor two. Therefore, for untrained users a higher level of guidance $\left(f_{\max }=4 \mathrm{~N}\right)$ gives better results, whereas trained users are less sensitive to the parameters of the method.

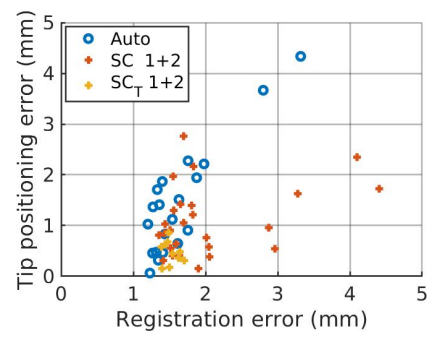

(a) In-plane positioning error

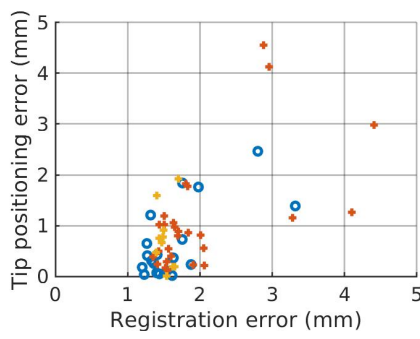

(b) Out-of-plane positioning error
Fig. 5. Needle tip positioning error with respect to the mean absolute registration error. Comparison between the Auto results, and the SC 1-2 results of the non-trained users $(\mathbf{S C})$ and the trained one $\left(\mathbf{S C}_{T}\right)$.

In the Fig. 5 are presented the needle tip placement error of the SC 1-2 methods, with respect to the registration error. Registration error is defined as the markers' positioning error after registration, averaged over the entire insertion. It is interesting to note that due to manipulations of the needle, untrained users tend to introduce additional registration errors during the insertion. Nevertheless, the resulting tip positioning error remains similar to the Auto method. Also, tip positioning error is lower than Auto for the same range of registration error thanks to the compensations of the users. More importantly, manipulations of the highly trained user does not introduce significant registration errors. Errors are consistent over the experiments and fit the clinical recommendations for such procedure for respectively $100 \%$ and $86 \%$ of the SC 1-2 trials for the highly trained user, and the untrained users, $20 \%$ and $7 \%$ using Manu, and up to $90 \%$ for Auto. Unfortunately, the number of trials per categories being low, statistical significance of the comparison between category cannot be guaranteed (except between the Man and the rest using the one-tailed Welch's t-test with $p<0.002$ ).

\section{CONCLUSION}

This paper presented a shared-control method allowing for percutaneous needle placement inside of soft tissue. The method allows the user to have full control over the tip position inside the tissue through a haptic device. The difficult needle steering required to follow the tip reference is done automatically. The user is guided with virtual fixtures keeping the needle on a pre-defined path. The method was tested and compared to a fully automated needle insertion and a manual insertion under fluoroscopic imaging.

The user study showed encouraging results showing that the method allows for a tip placement precision equivalent to an automated needle insertion for untrained users. Trained users are capable of aiming with more precision thanks to the visual feedback, reducing the in-plane precision. Further research would include a more populated user study.

Acknowledgement: This work was supported by French National Research Agency (ANR) within the project SPERRY ANR-18-CE33-0007 and the Investissements d'Avenir program (ANR-11-LABX-0004, Labex CAMI). 


\section{REFERENCES}

[1] R. Lencioni and L. Crocetti, "Radiofrequency Ablation of Liver Cancer," Techniques in Vascular and Interventional Radiology, vol. 10, no. 1, pp. 38-46, 2007.

[2] L. Crocetti, T. De Baere, and R. Lencioni, "Quality improvement guidelines for radiofrequency ablation of liver tumours," CardioVascular and Interventional Radiology, vol. 33, no. 1, pp. 11-17, 2010.

[3] B. B. Pua and C. T. Sofocleous, "Imaging to optimize liver tumor ablation," Imaging in Medicine, vol. 2, no. 4, pp. 433-443, 2010.

[4] R. S. Puijk, A. H. Ruarus, H. J. Scheffer, L. G. Vroomen, A. A. van Tilborg, J. J. de Vries, F. H. Berger, P. M. van den Tol, and M. R. Meijerink, "Percutaneous Liver Tumour Ablation: Image Guidance, Endpoint Assessment, and Quality Control," Canadian Association of Radiologists Journal, vol. 69, no. 1, pp. 51-62, 2018. [Online]. Available: https://doi.org/10.1016/j.carj.2017.11.001

[5] C. M. Hawkins, K. Kukreja, T. Singewald, E. Minevich, N. D. Johnson, P. Reddy, and J. M. Racadio, "Use of cone-beam CT and live 3-D needle guidance to facilitate percutaneous nephrostomy and nephrolithotripsy access in children and adolescents," Pediatric Radiology, vol. 46, no. 4, pp. 570-574, 2016.

[6] D. Jiao, Z. Zhang, Z. Sun, Y. Wang, and X. Han, "Percutaneous nephrolithotripsy: C-arm CT with 3D virtual navigation in non-dilated renal collecting systems," Diagnostic and Interventional Radiology, vol. 24 , no. 1 , pp. 17-22, 2018.

[7] T. L. De Jong, N. J. van de Berg, L. Tas, A. Moelker, J. Dankelman, and J. J. van den Dobbelsteen, "Needle placement errors: Do we need steerable needles in interventional radiology?" Medical Devices: Evidence and Research, vol. 11, pp. 259-265, 2018.

[8] P. Kulkarni, S. Sikander, P. Biswas, S. Frawley, and S. E. Song, "Review of Robotic Needle Guide Systems for Percutaneous Intervention," Annals of Biomedical Engineering, vol. 47, no. 12, pp. 2489-2513, 2019.

[9] O. Piccin, L. Barbé, B. Bayle, M. De Mathelin, and A. Gangi, "A force feedback teleoperated needle insertion device for percutaneous procedures," International Journal of Robotics Research, vol. 28, no. 9, pp. 1154-1168, 2009.

[10] T. Hiraki, T. Kamegawa, T. Matsuno, J. Sakurai, T. Komaki, T. Yamaguchi, K. Tomita, M. Uka, Y. Matsui, T. Iguchi, H. Gobara, and S. Kanazawa, "Robotic needle insertion during computed tomography fluoroscopy-guided biopsy: prospective first-in-human feasibility trial," European Radiology, vol. 30, no. 2, pp. 927-933, 2020.

[11] G. S. Fischer, I. Iordachita, C. Csoma, J. Tokuda, S. P. DiMaio, C. M. Tempany, N. Hata, and G. Fichtinger, "MRI-compatible pneumatic robot for transperineal prostate needle placement," IEEE/ASME Transactions on Mechatronics, vol. 13, no. 3, pp. 295-305, 2008.

[12] M. M. Arnolli, M. Buijze, M. Franken, K. P. de Jong, D. M. Brouwer, and I. A. Broeders, "System for CT-guided needle placement in the thorax and abdomen: A design for clinical acceptability, applicability and usability," International Journal of Medical Robotics and Computer Assisted Surgery, vol. 14, no. 1, 2018.

[13] Z. Han, K. Yu, L. Hu, W. Li, H. Yang, M. Gan, N. Guo, B. Yang, H. Liu, and Y. Wang, "A targeting method for robot-assisted percutaneous needle placement under fluoroscopy guidance," Computer Assisted Surgery, vol. 24, no. sup1, pp. 44-52, 2019.

[14] Z. He, K. W. Kwok, Z. Dong, G. Fang, J. D. L. Ho, C. L. Cheung, H. C. Chang, C. C. N. Chong, J. Y. K. Chan, and D. T. M. Chan, "Design of a Percutaneous MRI-Guided Needle Robot with Soft FluidDriven Actuator," IEEE Robotics and Automation Letters, vol. 5, no. 2, pp. 2100-2107, 2020.

[15] W. J. Heerink, S. J. Ruiter, J. P. Pennings, B. Lansdorp, R. Vliegenthart, M. Oudkerk, and K. P. de Jong, "Robotic versus Freehand Needle Positioning in CT-guided Ablation of Liver Tumors: A Randomized Controlled Trial," Radiology, vol. 290, no. 3, pp. 826-832, 2019.

[16] P. Li, Z. Yang, and S. Jiang, "Needle-tissue interactive mechanism and steering control in image-guided robot-assisted minimally invasive surgery: a review," Medical and Biological Engineering and Computing, vol. 56, no. 6, pp. 931-949, 2018.

[17] R. Alterovitz, K. Goldberg, J. Pouliot, R. Taschereau, and I. C. Hsu, "Sensorless Planning for Medical Needle Insertion Procedures," IEEE International Conference on Intelligent Robots and Systems, vol. 4, no. d, pp. 3337-3343, 2003.

[18] S. P. DiMaio and S. E. Salcudean, "Needle steering and model-based trajectory planning," Lecture Notes in Computer Science (including subseries Lecture Notes in Artificial Intelligence and Lecture Notes in Bioinformatics), vol. 2878, pp. 33-40, 2003.
[19] D. Glozman and M. Shoham, "Image-guided robotic flexible needle steering," IEEE Transactions on Robotics, vol. 23, no. 3, pp. 459-467, 2007.

[20] R. Kojcev, B. Fuerst, O. Zettinig, J. Fotouhi, S. C. Lee, B. Frisch, R. Taylor, E. Sinibaldi, and N. Navab, "Dual-robot ultrasound-guided needle placement: closing the planning-imaging-action loop," International Journal of Computer Assisted Radiology and Surgery, vol. 11, no. 6 , pp. $1173-1181,2016$

[21] Y. Adagolodjo, L. Goffin, M. De Mathelin, and H. Courtecuisse, "Robotic Insertion of Flexible Needle in Deformable Structures Using Inverse Finite-Element Simulation," IEEE Transactions on Robotics, vol. 35, no. 3, pp. 697-708, 2019.

[22] R. Alterovitz, M. Branicky, and K. Goldberg, "Motion planning under uncertainty for image-guided medical needle steering," International Journal of Robotics Research, vol. 27, no. 11-12, pp. 1361-1374, 2008.

[23] J. Chevrie, A. Krupa, and M. Babel, "Needle steering fusing direct base manipulation and tip-based control," Proceedings - IEEE International Conference on Robotics and Automation, vol. 2016-June, pp. 4450-4455, 2016.

[24] G. Lapouge, P. Poignet, and J. Troccaz, “Towards 3D ultrasound guided needle steering robust to uncertainties, noise and tissue heterogeneity," IEEE Transactions on Biomedical Engineering, pp. 1-1, 2020.

[25] D. A. Abbink, M. Mulder, and E. R. Boer, "Haptic shared control: Smoothly shifting control authority?" Cognition, Technology and Work, vol. 14, no. 1, pp. 19-28, 2012.

[26] M. Abayazid, C. Pacchierotti, P. Moreira, R. Alterovitz, D. Prattichizzo, and S. Misra, "Experimental evaluation of co-manipulated ultrasound- guided fl exible needle steering," no. May 2015, pp. 219230, 2016.

[27] M. Wartenberg, N. Patel, G. Li, and G. S. Fischer, "Towards synergistic control of hands-on needle insertion with automated needle steering for MRI-guided prostate interventions," Proceedings of the Annual International Conference of the IEEE Engineering in Medicine and Biology Society, EMBS, vol. 2016-Octob, pp. 5116-5119, 2016.

[28] J. Chevrie, A. Krupa, and M. Babel, "Real-time teleoperation of flexible beveled-tip needle insertion using haptic force feedback and 3d ultrasound guidance," Proceedings - IEEE International Conference on Robotics and Automation, vol. 2019-May, pp. 2700-2706, 2019.

[29] Y. Adagolodjo, L. Goffin, M. De Mathelin, and H. Courtecuisse, "Inverse real-time Finite Element simulation for robotic control of flexible needle insertion in deformable tissues," IEEE International Conference on Intelligent Robots and Systems, vol. 2016-Novem, pp. 2717-2722, 2016.

[30] P. Baksic, H. Courtecuisse, C. Duriez, and B. Bayle, "Robotic needle insertion in moving soft tissues using constraint-based inverse Finite Element simulation," IEEE International Conference on Robotics and Automation, p. 1, 2020.

[31] S. Miao, Z. J. Wang, Y. Zheng, and R. Liao, "Real-time 2D/3D registration via CNN regression," Proceedings - International Symposium on Biomedical Imaging, vol. 2016-June, pp. 1430-1434, 2016.

[32] K. Ohta, M. Shimohira, T. Murai, J. Nishimura, H. Iwata, H. Ogino, T. Hashizume, and Y. Shibamoto, "Percutaneous fiducial marker placement prior to stereotactic body radiotherapy for malignant liver tumors: An initial experience," Journal of Radiation Research, vol. 57, no. 2, pp. 174-177, 2016.

[33] Y. Adagolodjo, N. Golse, E. Vibert, M. De Mathelin, S. Cotin, and H. Courtecuisse, "Marker-Based Registration for Large Deformations Application to Open Liver Surgery," Proceedings - IEEE International Conference on Robotics and Automation, pp. 4007-4012, 2018.

[34] C. Duriez, C. Guébert, M. Marchal, S. Cotin, and L. Grisoni, "Interactive simulation of flexible needle insertions based on constraint models," Lecture Notes in Computer Science (including subseries Lecture Notes in Artificial Intelligence and Lecture Notes in Bioinformatics), vol. 5762 LNCS, no. PART 2, pp. 291-299, 2009. 